Rev. Elev. Méd. Vét. Pays trop., 1969, 22, 1 (17-23).

\title{
Possibilités et limites de la réaction d'inhibition de l'hémagglutination morbilleuse dans la sérologie de la Peste bovine (test IHM)
}

\section{III. - Utilisation du papier buvard dans la sérologie de la peste bovine mettant en jeu le test IHM}

\author{
par Y. MAURICE ef A. PROVOST
}

(l. E. M. V.T., Laborataire de Recherches Vérérınaires de Farcha, Fort-Lamy, Tchad)

\begin{abstract}
RÉSUMÉ
L'absorption des sérums à éprouver sur une bande de papier buvard permer de surmonter la difficulté de l'envo des échantillons au laboratoıre en vue du dıagnostic sérologique de peste bovine. Une quantıé donnée de sérum est absorbée sur une surface délermınée de papıer buvard. L'épreuve d'ınhibition de l'hémagglutination morbılieuse (lest IHM) se fait au laboratore en reconstituant le sérum à éprouver.
\end{abstract}

\section{INTRODUCTION}

Les méthodes de base du diagnostic de la peste bovine au Tchad sont la précipitation diffusion en gélose à partır du ganglıon suspect en présence d'un sérum hyperimmun précıpitant de référence, l'isolement du virus sur cellules de reins d'embryon de veau et son identification, et l'inhibition de l'hémagg/utınation morbilleuse, à partir du sérum suspect, qui vise à mettre en évidence l'apparition d'antıcorps ou une montée du taux de ceux-cl. En présence de souches hypovirulentes qui ne tuent que peu ou pas de bovins, cette dernıère technıque se montre très utile. C'est celle-ci que l'on a utılisée depuss tros années parce que, il faut bien le dire aussi, le sérum est souvent le seul prélèvement reçu au laboratoire pour établir les diagnostıcs de peste bovine.

L'envoi rapide au laboratorre des échantillons de sérums recueillis sur le terraın afin de diagnostiquer la peste bovine, constitue d'une façon générale un sérieux problème dans de nombreux pays par suite des difficultés de transport et de la nécessité du maintien sous froid des sérums. Il arrive en effet souvent, surtout en saison chaude, que les échantilions soient altérés au cours du voyage, ce qui nuit à la spécificité de la réaction au laboratoire. Une méthode par laqueile les sérums à éprouver sont absorbés sur papier buvard, a été étudiée et mise au point pour surmonter ces difficultés. L'absorption des sérums sur papier buvard ou papıer filtre a déjà suscıté un certaın nombre de travaux : elle a été utılısée par ADAMS et HANSON (1) pour la stomatite vésiculeuse, KARSTAD et coll. (9) pour l'encéphalıte équine de l'Est, GREEN et OPTOM (8) pour la polyomyélite, WORTH (14) pour les orellons, BENDSON et MICKLE (3) pour la maladie de Carré, BRODY et coll. (6) pour les adénovirus et la rougeole, GAGGERO (7) pour la fièvre aphteuse.

Il était intéressant, dans un pays où les trans- 
ports sous glace posent un problème sérieux, d'étudıer la possıbılıté d'utiliser le sérum desséché sur papier buvard pour le diagnostic de peste bovine mettant en jeu le test IHM.

\section{MATÉRIEL ET MÉTHODE}

\section{— Sérums.}

Les échantillons de sérum ef de sang proviennent de bovins vaccinés contre la peste bovine et abattus à l'abattoir de Farcha ef d'anımaux d'expérience. Les échantillons de sérum utılısés proviennent également des envois adressés au laboratoire de Farcha ces deux dernières années en vue d'établir un diagnostıc de peste bovine. Les sérums contrôlés sont les sérums correspondant à ceux absorbés sur papier buvard et conservés à $-15^{\circ} \mathrm{C}$.

\section{- Papier buvard.}

Le papier buvard est celui vendu couramment dans le commerce à Fort-Lamy (papier buvard pesant $1,35 \mathrm{~g} \mathrm{par} \mathrm{dm}^{2}$, de couleur blanche). Ces papiers buvards ne sont pas stérilisés et aucune précaution particulière n'est prise pour éviter les contaminations. Seul le papier buvard a été utilssé parce qu'un certain nombre d'auteurs, VAISMAN ef coll. (13) et OPPELAR (12) en particulier on ${ }^{\dagger}$ obtenu des résultats meilleurs qu'avec les papiers filtres.

\section{- Manipulations sur le sang.}

Chaque échantıllon de sang est absorbé par une surface déterminée de papier buvard. II est identifié au moyen d'un numéro inscrit avec un crayon norr ordinaire à l'envers du papier comportant également lo date de prélèvement du sang. Le papier buvard utilisé retıenf $7 \mathrm{ml}$ de sang sur une surface de $6 \times 20 \mathrm{~cm}$. Après imprégnation par le sang, les bandes sont mıses à sécher, suspendues par une extrémité, une demi-heure à 1 heure à température ordınaıre, puis conservées sous enveloppe. Avant utilisation, chaque bande est divisée en 2 et l'on place une de ces moitıés dans un tube de $22 \mathrm{~mm}$ de diamètre stérile où sera faite la reconstitution.

Pour chaque demi-bande de papier on ajoute dans le tube $7 \mathrm{ml}$ de tampon de Cohen de façon à avoir une reconstitution à la dilution de 1/2 de volume initial de sang absorbé. L'ensemble buvard-tampon est placé d̀ $4^{\circ} \mathrm{C}$ pendant une nult ou simplement une heure à la température ordinaıre (les résultats sont identıques).

\section{- Manipulations sur le sérum.}

Chaque échantıllon de sérum est absorbé sous le volume de $1 \mathrm{ml}$ sur une bande de papier buvard de $4 \mathrm{~cm} \times 12,5 \mathrm{~cm}$. Chaque échantillon est identifié comme précédemment. Après imprégnation par le sérum, les bandes sont mises à sécher $1 / 2$ heure d̀ 1 heure à température ord naire puis conservées sous enveloppe, pendant des temps variables, à la température ordinaire, à $4{ }^{\circ} \mathrm{C}$ et à $-15^{\circ} \mathrm{C}$. Certains échantillons sont laıssés au contact de l'air et de la lumière. Avant utilisation, chaque bande est placée dans un tube de $22 \mathrm{~mm}$ de diamètre, stérıle, ef on reconstitue avec $2 \mathrm{ml}$ de tampon de Cohen pour avar une dilution au 1/2. Après un contact de 1 heure à température ordinaire ou de une nuit à $4{ }^{\circ} \mathrm{C}$, le papier buvard est pressé contre le fond du tube avec 1 baguette de verre et l'on obtıent $0,6 \mathrm{ml}$ de sérum reconstitué au 1/2, quantité suffisante pour effectuer le test IHM. II n'a pas été jugé utile de recourir à l'extraction du sérum par la méthode d'ANDERSON et coll. (2) ou de OPPELAR (11), l'extraction simple donnant satisfaction.

\section{- La réaction.}

La réaction d'inhibition de l'hémagglutination morbilleuse est effectuée à partır des sangs ou des sérums reconstitués en utılisant la même technique que celle mise en ceuvre pour la réaction d'inhibition de l'hémagglutination à partir du sérum lui-même $(4,5)$. Les limites d'interprétation de la réaction, ses limites d'emploi, sont également celles énumérées dans les articles précédents (10) lorsque l'on utilise le sérum lui-même.

\section{RÉSULTATS}

Les résultats mentionnés ci-dessous concernent des réactions où les sérums absorbés et les sérums témoıns ont été testés avec le même lot d'antigène et d'hématıes de patas, dans les mêmes conditions expérimentales.

\section{$1^{\circ}$ A partir du sang :}

a) Sang de bovins vaccinés avec un vaccin antipestique de culture cellulare ou un vaccin 
caprinisé. Les animaux ont été vaccinés une ou plusieurs fols.

Le détail des résultats est le sulvant:

- Nombre de sérums positıfs au test IHM : 25 sur 86.

- Nombre de sangs reconstitués 8 jours après absorption et positıfs au test IHM : 14 sur $86(*)$.

- Nombre de réactions illısıbles à partır du sang : 22 sur 86.

Conclusion : Il n'est pas possible d'utiliser le sang récolté directement sur papier buvard dans la sérologie de la peste bovine mettant en jeu la réaction d'inhibition de l'hémagglutınation morbilleuse en raison :

- Du nombre élevé de réactions Illısıbles et de réactions difficilement lisibles aux dilutions $1 / 2$ et $1 / 4$ (hémolyse des globules rouges absorbés sur le papier buvard qui rend le liquide de reconstitution rouge sombre).

- De la coagulation fréquente du liquide de reconstitution quand celui-ci est inactıvé à $56^{\circ} \mathrm{C}$.

- Des résultats peu fidèles obtenus.

\section{$2^{\circ}$ A partir du sérum :}

a) Comportement des sérums absorbés sur papier buvard et conservés à température ordinaire et à la lumière, à température ordınaire et à l'abri de la lumière, à $4^{\circ} \mathrm{C}$ à l'abri de la lumière.

- 33 sérums envoyés au laboratoire pour diagnostic de peste bovine ont été mis sur papier buvard en quatre exemplaires.

- 1 lot a été conservé à température ordınaıre. à la lumière,

- 2 lots ont été conservés à température ordinaire, à l'abrı de la lumière,

- 1 lot a été conservé à $4^{\top} \mathrm{C}$.

La réaction IHM est effectuée 60 jours plus tard en utilısant les sérums reconstıtués extemporanément et le sérum contrôle correspondant. Les résultats sont mentionnés dans le tableau $\mathrm{l}$.

La même opération a été effectuée sur 28 sérums envoyés au laboratorre pour diagnostic de peste bovine. $\mathrm{La}$ réaction $\mathrm{IHM}$ a été effectuée 2 mois et demi après l'absorption.

(*) Les sérums correspondants à 2 de ces sangs sont négatifs au test IHM
Les résultats obtenus sont mentionnés dans le tableau 11.

Les 61 sérums précédents placés sur papier buvard et conservés à température ordinaire sous enveloppe ont également été testés 15 jours après I'absorption. Les taux d'anticorps IHM sont identıques à ceux des témoins.

Conclusion : après un délai de 60 jours, les anticorps IHM sont rendus inactifs à température ordinaıre et à la lumière ; Ils sont également inactivés pour la plupart à l'abri de la lumière et à température ordinaıre. Ils persistent d̀ un taux identıque après un séjour de 15 jours à température ordinaire, sous enveloppe, d̀ l'obscurité et après 2 mois et demi de séjour à 4 o $C$, sous enveloppe.

b) Comportement des sérums absorbés sur papier buvard ef conservés à 4 o $C$, à l'abrı de la lumière.

a) Sérums d'animaux malades ou convalescents de peste bovine :

- 9 sérums mis sur buvard possédalent 8 jours, 15 jours et 30 jours après l'absorption le même titre d'antıcorps IHM que les sérums témains.

- 9 sérums mis sur buvard possédaient 1 mois et 10 jours après l'absorption le même titre d'anticorps IHM que les sérums témoins.

- 14 sérums mis sur buvard possédaient 50 jours après l'absorption le même titre d'anticorps IHM que les sérums témoins.

- Les 33 sérums de l'expérience mentionnée précédemment (tableau 1) testés 60 jours après l'absorption possédaient également le même titre d'anticorps IHM que les sérums témoins.

- Les 28 sérums de l'expérience mentionnée précédemment (tableau II) testés 2 mois et demı après l'absorption se sont comportés comme les sérums témoins.

B) Sérums d'anımaux vaccinés :

- 73 sérums d'animaux vaccinés ont montré 1 mois après l'absorption le même titre d'anticorps IHM que les sérums témoins.

- 21 sérums d'animaux vaccinés ont montré 8 jours et 1 mols après l'absorption le même taux d'anticorps IHM que les sérums témoins.

- 10 sérums d'anımaux vaccınés ont montré 3 mols el demı après l'absorption une baisse du titre d'anticorps IHM. La différence est encore 
TABLEAU $\mathrm{N}^{\circ} \mathrm{I}$

Influence du temps, de la tempêrature et de la lumière sur la conservation des anticarps IFM dans les êchantillons de sérums rêcoltés sur papier buvard.

\begin{tabular}{|c|c|c|c|c|c|}
\hline $\begin{array}{l}\mathrm{N}^{\circ} \\
\text { des } \\
\text { sérums }\end{array}$ & $\begin{array}{c}\text { Sërum témoin } \\
\text { conservê à } \\
15^{\circ} \mathrm{C} .\end{array}$ & $\begin{array}{l}15 \text { jours à } \\
\text { température } \\
\text { oxdinaire }\end{array}$ & $\begin{array}{r}60 \mathrm{~J}: \text { à } 4^{\circ} \mathrm{C} \\
\text { à I abri de } \\
\text { la Iurière }\end{array}$ & $\begin{array}{l}60 \mathrm{~J} \text { à l'abri } \\
\text { de la lumiêre } \\
\text { a température } \\
\text { ordinaire }\end{array}$ & $\begin{array}{l}60 \mathrm{~J} \text {, à la } \\
\text { lumière } \\
\text { température } \\
\text { ordinaíre }\end{array}$ \\
\hline 1 & $+1 / 8$ & $+1 / 8$ & $+1 / 8$ & traces $1 / 2$ & - \\
\hline 2 & $+1 / 2$ traces $1 / 4$ & $+2 / 2$ & $+1 / 2$ traces $1 / 4$ & - & - \\
\hline 3 & $+1 / 8$ & $+1 / 8$ & $+1 / 8$ & - & - \\
\hline 4 & - & - & - & - & - \\
\hline$s$ & $+1 / 16$ & $+1 / 16$ & $+1 / 16$ & - & - \\
\hline 6 & $+1 / 8$ & $+1 / 8$ & $+1 / 8$ & - & - \\
\hline 7 & $+1 / 2$ traces $1 / 4$ & $+1 / 2$ traces $1 / 4$ & $+1 / 2$ traces $1 / 4$ & - & - \\
\hline 8 & $+1 / 4$ & $+1 / 4$ & $+1 / 4$ & traces $1 / 2$ & - \\
\hline 9 & $+1 / 8$ & $+1 / 8$ & $+1 / 8$ & - & - \\
\hline 10 & $+1 / 8$ & $+1 / 8$ & $+1 / 8$ & - & - \\
\hline 11 & $+1 / 16$ & $+1 / 16$ & $+1 / 16$ & - & - \\
\hline 12 & - & - & - & - & - \\
\hline 13 & - & - & - & - & - \\
\hline 14 & - & - & - & - & - \\
\hline 15 & $+1 / 16$ & $+1 / 16$ & $+1 / 16$ & Cassé & - \\
\hline 16 & - & - & - & - & - \\
\hline 17 & - & - & - & - & - \\
\hline 18 & $+1 / 4$ & $+1 / 4$ & $+1 / 4$ & - & - \\
\hline 19 & - & - & - & - & - \\
\hline 20 & - & - & - & - & - \\
\hline 21 & $+1 / B$ & $+1 / 4-50 \mathrm{p} \cdot 1001 / 8$ & $+1 / 8$ & - & - \\
\hline 22 & - & - & - & - & - \\
\hline 23 & $+1 / 8$ & $+1 / 8$ & $+1 / 8$ & - & - \\
\hline 24 & - & - & - & - & - \\
\hline 25 & $+1 / 8$ & $+1 / 8$ & $+1 / 8$ & - & - \\
\hline 26 & - & - & - & - & - \\
\hline 27 & $+1 / 8$ & $+1 / 8$ & $+1 / 8$ & - & - \\
\hline 28 & $+1 / 8$ & $+1 / 8$ & $+1 / 8$ & - & - \\
\hline $\begin{array}{l}29 \\
30\end{array}$ & $+\overline{1 / 32}$ & $+\overline{1 / 32}$ & $+\overline{1 / 32}$ & - & - \\
\hline 31 & - & - & - & - & - \\
\hline 32 & - & - & - & - & - \\
\hline 33 & - & - & - & - & - \\
\hline
\end{tabular}


TABLEAU N* II

Influence du temps, de la température et de la lumière sur la conservation des anticorps IHM dans les échantillons de sérums récoltês sur papier buvard.

\begin{tabular}{|c|c|c|c|c|}
\hline \multirow{2}{*}{$\begin{array}{c}\mathrm{N}^{*} \\
\text { des } \\
\text { sérums }\end{array}$} & \multirow{2}{*}{$\begin{array}{c}\text { Sêrums témoins } \\
\text { conservés } \\
\text { à }-15^{\circ} \mathrm{C} .\end{array}$} & \multicolumn{3}{|c|}{ Echantillons conservés pendant 2 mois et demi } \\
\hline & & $\begin{array}{c}\text { à la température } \\
\text { de } 4^{\circ} \mathrm{C} .\end{array}$ & $\begin{array}{l}\text { à la température } \\
\text { amb1ante et sous } \\
\text { enveloppe }\end{array}$ & $\begin{array}{c}\text { à la température } \\
\text { ambiante et à la } \\
\text { lumière }\end{array}$ \\
\hline 1 & Traces $1 / 2$ & Traces $1 / 2$ & - & - \\
\hline 2 & $+1 / 8$ & $+1 / 8$ & - & - \\
\hline 3 & $+1 / 4$ & $+1 / 4$ & - & - \\
\hline 4 & $+1 / 2$ & $+1 / 2$ & - & - \\
\hline 5 & $+1 / 8$ & $+1 / 8$ & - & - \\
\hline 6 & - & - & - & - \\
\hline 7 & Traces $1 / 2$ & Traces $1 / 2$ & - & - \\
\hline 8 & Traces $1 / 2$ & Traces $1 / 2$ & - & - \\
\hline 9 & $+1 / 4$ & $+1 / 4$ & - & - \\
\hline 10 & $+1 / 4$ & $+1 / 4$ & - & - \\
\hline 11 & - & - & - & - \\
\hline 12 & $+1 / 2$ & $+1 / 2$ & - & - \\
\hline 13 & Traces $1 / 2$ & Traces $1 / 2$ & - & - \\
\hline 14 & - & - & - & - \\
\hline 15 & - & - & - & - \\
\hline 16 & - & - & - & - \\
\hline 17 & $+1 / 8$ & $+1 / 8$ & $+1 / 8$ & - \\
\hline 18 & - & - & - & - \\
\hline 19 & $+1 / 16$ & $+1 / 16$ & - & - \\
\hline 20 & - & - & - & - \\
\hline 21 & $+1 / 8$ & $+1 / 8$ & - & - \\
\hline 22 & - & - & - & - \\
\hline 23 & $+1 / 8$ & $+1 / 8$ & - & - \\
\hline 24 & $+1 / 4$ & $+1 / 4$ & - & - \\
\hline 25 & $+1 / 4$ & $+1 / 4$ & - & - \\
\hline 26 & - & - & - & - \\
\hline 27 & - & - & - & - \\
\hline 28 & - & - & - & - \\
\hline
\end{tabular}

plus significative 5 mols et 8 jours après (tableau III).

Conclusion : un séjour de 6 semaines à $4^{\circ} \mathrm{C}$ ne détruit pas les anticorps IHM contenus dans les sérums absorbés sur papier buvard. $\mathrm{Cec}$ n'est plus vrai après 14 semaınes et au-delà.

c) Comportement des sérums absorbés sur papier buvard et conservés à $-15^{\circ} \mathrm{C}$.

Les expériences ont été réalısées en utılısant des sérums d'animaux convalescents ou malades de peste bovine. 9, 14 et 30 sérums ont été analysés avec le test $\mathrm{HHM}$, respectivement 40 ,
50 et 63 jours après l'absorption. Les titres obtenus au bout de ces délais sont les mêmes que ceux des sérums témoıns.

Conclusion : pendant les 6 premières semaines les anticorps IHM ne sont pas détruits lorsque le papier buvord est conservé à $-15^{\circ} \mathrm{C}$.

\section{CONCLUSION}

10 Le papier buvard conserve les anticorps IHM. Ils sont d'autant mieux préservés que les papiers buvards sont placés aussitôt au froid et 
TABLEAU $N^{0}$ III

Influence du temps sur la conservation des anticorps IHM.

\begin{tabular}{|c|c|c|c|}
\hline \multirow{2}{*}{$\begin{array}{l}\mathbb{N}^{\circ} \\
\text { des } \\
\text { Sêrums }\end{array}$} & \multirow{2}{*}{$\begin{array}{l}\text { Sérum témoin } \\
\text { conservé à }-15^{\circ} \mathrm{C} \text {. }\end{array}$} & \multicolumn{2}{|c|}{ Paplers buvards conservês à $4^{\circ} \mathrm{C}$. } \\
\hline & & Pendant 3 mois $1 / 2$ & $\begin{array}{c}\text { Pendant } 5 \text { mois et } \\
8 \text { jours }\end{array}$ \\
\hline 1 & $+1 / 8$ & $+1 / 4$ & - \\
\hline 2 & $+1 / 16$ & $+1 / 8$ & - \\
\hline 3 & $+1 / 8$ & $+1 / 4$ & - \\
\hline 4 & $+1 / 8$ - Traces $1 / 16$ & $+1 / 4$ & $+1 / 2$ \\
\hline 5 & $+1 / 16$ & $+1 / 8$ & R.N.E. \\
\hline 6 & $+1 / 4$ & - & - \\
\hline 7 & $+1 / 8$ & $+1 / 4$ & - \\
\hline 8 & $+1 / 4$ & - & - \\
\hline 9 & $+1 / 32$ & $+1 / 16$ & $+1 / 4$ \\
\hline 10 & $+1 / 8$ & $+1 / 4$ & - \\
\hline
\end{tabular}

R.N.E. = Réaction non effectuée,

à l'abri de la lumière. En effet, la chaleur ef la lumière dénaturent au bout d'un certain temps ces anticorps et gênent airsı l'obtention de réaction IHM positive ou en modifient le résultat quantitatif. Les échantillons de sérums absorbés sur papier buvard devront donc être conservés sous enveloppe sombre, à $-15^{\circ} \mathrm{C}$ ou à $4{ }^{\circ} \mathrm{C}$. et devront être utilısés pour le test IHM dans les deux mois qui suivent l'absorption.

$2^{\circ}$ Ce procédé de récolte des sérums pour envoi d'échantilions au laboratore, en particulier en vue du dıagnostic de peste bovine est particulièrement intéressant pour diverses ralsons :
- disparition des risques de casse des flacons et par conséquent des pertes de sérum :

- rapidité et économie des envois par la poste, sous enveloppe ;

- moindre encombrement des freezers et frigıdaires.

3o II va de soi que la méthode dat être standardisée. II appartient au laboratore de tester le papier à utıliser, c'est-à-dire d'éliminer les papiers buvards couleur, de déterminer leur coefficient d'absorption que l'on pourrast définir par la surface exprimée en $\mathrm{cm}^{2}$, nécessatre pour absorber $1 \mathrm{ml}$ de sérum. Les papiers buvards seralent ainsi IIvrés « prêts pour emplor » accompagnés d'une notıce explicative.

\section{SUMMARY}

Possibilities and lımıts of the measles haemagglutination inhibition test in the serology of rinderpest.

\section{Use of blotting paper in the serology of rinderpest involving $\mathrm{MHI}$ test}

The difficulties resulting from dispatch of samples to laboratory for the serological diagnosis of Rinderpest can be avoided by using the absorption of serum to be tested on blotting paper strip. A known quantily of serum is absorbed on a known surface of blotting paper. The measles haemagglutination inhibition test is carried out in laboratory by reconstituting the serum to be fested. 


\section{RESUMEN}

Posıbilidades y lımiles de la reacción de inhibıción de la hemaglutinación morbillosa en la serologia de la peste bovina.

III. Utılización del papel secante en la serologia

de la peste bovina empleando la prueba IHM

Se pueden evitar las dificultades del envió de las muestras al laboralorio para el diagnóstico serologico de la peste bovina utilizando la absarpción de los sueros a probar sobre una lıra de papel secante. Una superficie determınada de papel secante absorbe una cantidad conocida de suero Se hace en el laboratorio la prueba de inhibición de la hemaglutinación morbıllosa (prueba $\mathrm{IHM}$ ) reconstituyendo el suero a probar.

\section{RÉFÉRENCES}

1. ADAMS (E.) et HANSON ( $R$ P.). - A procedure for adsorbing virus neutralizing antibodies on paper discs. Journ. Bact., 1956, 72,572 .

2. ANDERSON (R. I.), SADUN (C. H.) et WILLIAMS (」. S.). - A technique for the use of minute amounts of dried blood in the fluorescent antibody test for schistosomiasis. Exp. Parast., 1961, 11 : 111-116.

3. BENSON (T.F.) et MICKLE (E.). - A filter paper disc method for collection of blood simples for serological procedures. Cornell Vet., 1964, $54: 331$.

4. BOGEL (K.), ENDERS-RUCKLE (G.) et PROVOST (A.). - Une réaclion sérologique rapide de mesure des anticorps antibovipestiques. C. R. Acad. Sci. Poris, 1964, 259 : 482.

5. BOGEL (K.), PROVOST (A.) et ENDERSRUCKLE (G.). - Hemmagglutinations Hemmungsreaktion mit Masernantigen bel Rinderpest. I. Anwendung in der Diagnostik. II. Antikörperproduktion nach Inokulation Verschiedener Lebendimpstoffe beım Rind. Zentbl. Bakt. 1. (Org.), 1966, $199: 1-19$ et $201: 137-152$.

6. BRODY (J. A.), MC ALISTER (R.) et HASELEY (R.) et LEE (P.). - Use of dried whole blood collected ou filter paper disc in adenovirus complement fixation and measle hemagglutination inhibition test. J. Immunology, 1964, $92: 854$.
7. GAGGERO (A.) et SUTMOLLER (P.). The use of serum and blood direct on blotting paper in the detection of foot and mouth disease antibody. Bri. Vet. J., 1965, 121, 509514.

8. GREEN (R. H) et OPTON (E. M.). - Amer. J. Hyg., 1960, 72, 195.

9. KARSTAD (L.), SPALATIN (J.) et HANSON (R. P.). - Application of the paper disc technic to the collection of whole blood and serum samples in studies of eastern equine encephalomyelitis. J. Infect. Dis., 1957, 101, 295-299.

10. MAURICE (Y.), PROVOST (A.) et BORREDON (C.). - Possibilités et limites de la réaction d'inhıbition de l'hémagglutination morbilleuse dans la peste bovine (test IHM). I. Interprétation et utilité de la réaction. Rev. Elev. Méd. Vét. Poys trop., 1969, 22, $1: 1-8$.

11. OPPELAR (L.). - Simplified techniques for the extraction of blood and serum from filter paper. Trop. Geog. Med., 1966, 18, 67-70.

12. OPPELAR (L.). - The use of filter paper as a transport medium for blood and serum. Trop. Geog. Med, 1966, 18 : 60-66.

13. VAISMAN (A.), HAMELIN (A.) et GUTHE (T.). - La technique des anticorps fluorescents pratiquée sur sang desséché et élué. Comparaison avec le F. T. A., le $T$. P. I. el les épreuves à l'antigène lipidique pratiquées sur le sérum. Bull. de l'O. M. S., 1963, $29: 1$

14. WORTH (R. M.). - Amer. Journ. Hyg. $1963,78,349$. 